\title{
Chain breaking and Kosterlitz-Thouless scaling at the many-body localization transition in the random-field Heisenberg spin chain
}

\author{
Nicolas Laflorencie, ${ }^{1, *}$ Gabriel Lemarié $\odot,{ }^{1,2,3, \dagger}$ and Nicolas Macé $\odot^{1, \ddagger}$ \\ ${ }^{1}$ Laboratoire de Physique Théorique, IRSAMC, Université de Toulouse, CNRS, UPS, France \\ ${ }^{2}$ MajuLab, CNRS-UCA-SU-NUS-NTU International Joint Research Unit, Singapore \\ ${ }^{3}$ Centre for Quantum Technologies, National University of Singapore, Singapore
}

(Received 27 April 2020; revised 3 November 2020; accepted 4 November 2020; published 25 November 2020)

\begin{abstract}
Despite tremendous theoretical efforts to understand subtleties of the many-body localization (MBL) transition, many questions remain open, in particular concerning its critical properties. Here we make the key observation that MBL in one dimension is accompanied by a spin freezing mechanism which causes chain breaks in the thermodynamic limit. Using analytical and numerical approaches, we show that such chain breaks directly probe the typical localization length and that their scaling properties at the MBL transition agree with the Kosterlitz-Thouless scenario predicted by phenomenological renormalization group approaches.
\end{abstract}

DOI: 10.1103/PhysRevResearch.2.042033

Introduction. The field of interacting quantum systems in the presence of disorder has attracted a lot of attention over the past two decades. Besides tremendous theoretical efforts [1-11], a growing number of experimental realizations have also emerged, based on ultracold atoms or trapped ions [12-16], superconducting qubits [17-19], and solid-state setups [20-22]. Building on this collective movement (for recent reviews, see Refs. [7-11]), it is currently well admitted that upon increasing disorder several low-dimensional quantum interacting systems can undergo a transition toward a many-body localized (MBL) phase. This nonergodic regime is reasonably well characterized, mostly by exact diagonalization (ED) techniques [23,24] showing various properties of MBL states: Poisson spectral statistics, area-law entanglement, emerging integrability, logarithmic spreading of entanglement, and eigenstate multifractality. While typically limited to $L \approx 20$ interacting two-level systems (due to exponentially small level spacings $\propto 2^{-L}$ at high energy), ED studies have nevertheless showed a clear ergodicity breaking transition for the random-field spin-1/2 Heisenberg chain model

$$
\mathcal{H}=\sum_{i=1}^{L}\left(\vec{S}_{i} \cdot \vec{S}_{i+1}-h_{i} S_{i}^{z}\right),
$$

where $h_{i}$ are independently drawn form a uniform distribution $[-h, h]$. Despite recent debates [25-31], $h_{c} \approx 4$ is the most

\footnotetext{
*nicolas.laflorencie@irsamc.ups-tlse.fr

†lemarie@irsamc.ups-tlse.fr

"nicolas.mace@ irsamc.ups-tlse.fr
}

Published by the American Physical Society under the terms of the Creative Commons Attribution 4.0 International license. Further distribution of this work must maintain attribution to the author(s) and the published article's title, journal citation, and DOI. accepted numerical estimate [5,23] for the critical disorder strength at infinite temperature.

A more serious issue concerns the universality class of the ergodic-MBL transition, for which numerical simulations yield a correlation length $\xi \sim\left|h-h_{c}\right|^{-v}$ with an exponent $v \approx 1$ for both sides of the transition [23,29,32-35]. Only recently, a finite-size scaling analysis of the multifractal properties has found an asymmetric criticality for the multifractal dimension [36], thus making an interesting connection with the Anderson problem on random graphs [37-50] where such unusual scaling properties were first found $[45,49]$. Nevertheless, most numerical studies violate the Harris bound [51,52], and a fully consistent finite-size scaling theory is still missing [53].

In such a puzzling context, progress has been made to build an analytical theory able to describe the ergodicMBL transition [54-62]. The most successful description, based on the so-called "avalanche" scenario [63,64], proposes a phenomenological renormalization group (RG) treatment, working in the MBL regime where there are large insulating blocks compete with small ergodic inclusions. In this framework, recent works [65-67] found a RG flow of the Kosterlitz-Thouless (KT) form. The MBL phase is described as a line of fixed points with a vanishing density of ergodic inclusions and a finite typical localization length $\zeta$, which controls the spatial extension of the $l$ bits [68-70]. When the delocalization transition is approached from the MBL side, the typical localization length $\zeta$ grows and reach a finite critical value $\zeta_{c}=(\ln 2)^{-1}$ at the transition point $h_{c}$, with a singular behavior [71]

$$
\zeta^{-1}-\zeta_{c}^{-1} \propto \sqrt{h-h_{c}}
$$

As argued in Ref. [66], a diverging length scale $\sim \exp \left(c / \sqrt{h-h_{c}}\right)$ should control finite-size effects, thus explaining some limitations in the numerics. 

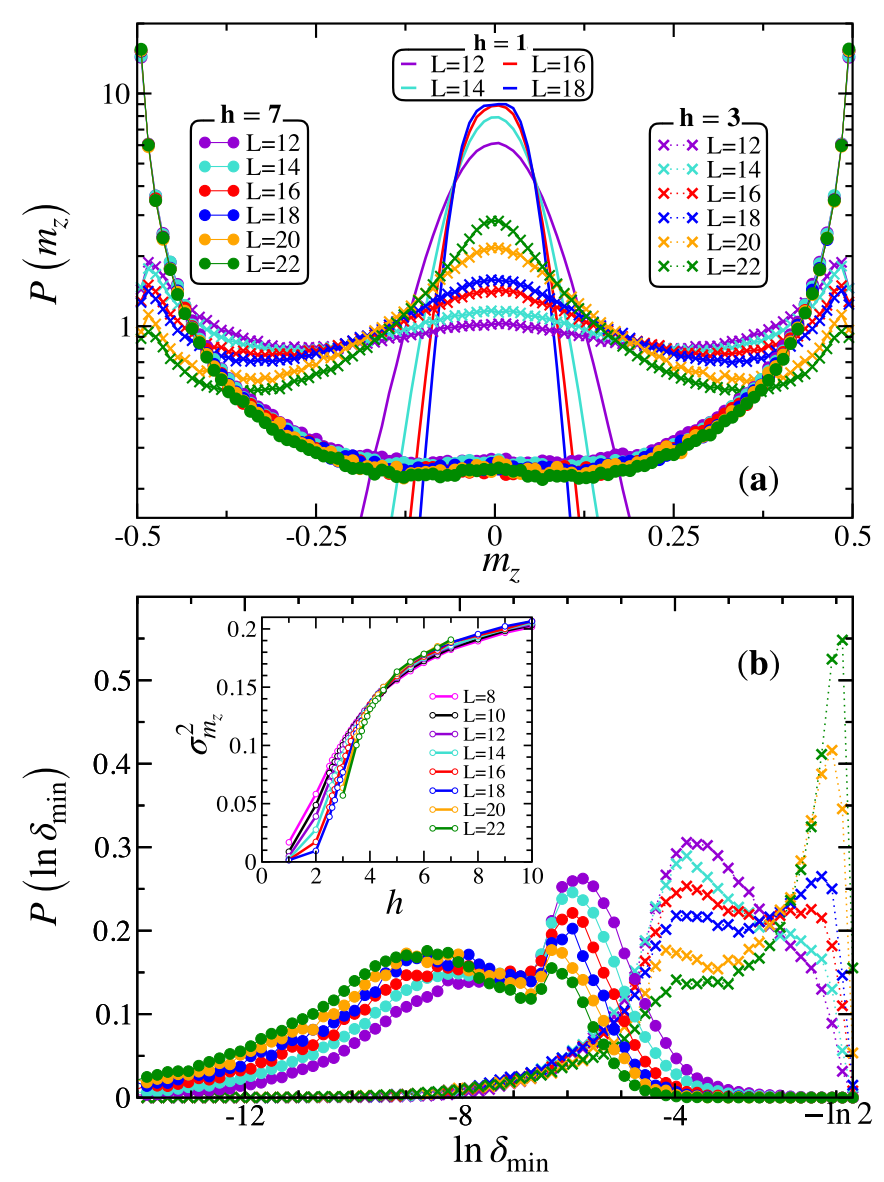

FIG. 1. Histograms of (a) the local magnetizations $m_{z}=\left\langle S_{i}^{z}\right\rangle$ displayed for $h=1,3,7$ and (b) of the maximally polarized sites Eq. (4). Inset: Variance $\sigma_{m_{z}}^{2}$ of the local magnetization plotted as a function of $h$ for various lengths $L$. Shift-invert ED results for infinite-temperature eigenstates of Eq. (1), performed over thousands of independent random samples.

In this Rapid Communication, we show that one can overcome such finite-size constraints by measuring spin polarization, a simple local observable of the $S=1 / 2$ Hamiltonian Eq. (1). Our main finding is that the extreme statistics of the polarizations gives direct access to the typical MBL localization length $\zeta$, which shows a very good agreement with Eq. (2). We numerically find finite-size effects of KT type, controlled by a correlation length $\sim \exp \left(c /\left(h-h_{c}\right)^{\nu_{\text {loc }}}\right)$, with $v_{\text {loc }} \simeq 0.5$. Building on Refs. [36,45,49], a careful scaling analysis of our ED data reveals that they are best described by a volumic scaling variable $\mathcal{N} / \Lambda$ in the delocalized regime, where $\mathcal{N}$ is the Hilbert-space size and $\Lambda$ is a nonergodicty volume, while a logarithmic scaling variable $(\ln L) / \lambda$ dictates the behavior in the MBL regime, with $\lambda^{-1} \propto \zeta^{-1}-\zeta_{c}^{-1}$ following the square-root KT singularity Eq. (2).

Distribution of local polarizations and extreme value statistics. Let us start the discussion by looking at the local polarizations $m_{z}=\left\langle S^{z}\right\rangle$, computed for individual infinite-temperature eigenstates using ED simulations for the Hamiltonian Eq. (1). As already noticed in Refs. [72-75], the histograms $P\left(m_{z}\right)$ display distinct features across the two regimes, as illustrated in Fig. 1(a) for three representative values of the disorder strength $(h=1,3,7)$. At weak disorder, we expect from the eigenstate thermalization hypothesis (ETH) [76-78] to observe Gaussian distributions, peaked around $m_{z}=0$ and shrinking with increasing system size, as clearly visible for $h=1$. At $h=3$, a more complex form emerges with deviations from Gaussianity [79,80]: Strongly polarized $\left(m_{z} \simeq\right.$ $\pm 1 / 2$ ) sites appear, but their density shrinks down with system size (as evidenced by the decrease of magnetization variance; see inset). At strong disorder $h=7$, the density of strongly polarized sites no longer shrinks down: ETH is violated and the distribution is U-shaped, almost free of finite-size effects.

To quantify this effect, we introduce the deviation from perfect polarization $\delta=\frac{1}{2}-\left|m_{z}\right|$. While $\delta>0$ for finite systems, $\delta$ becomes arbitrarily small for large disorder, as evidenced in Fig. 1(a) at $h=7$ where one observes [81]

$$
P(\delta) \propto \delta^{-1+\frac{1}{\gamma}} \quad(\delta \rightarrow 0),
$$

with an exponent $\gamma \geqslant 0$ related to extreme value statistics [82], as we discuss now. In each finite sample, we define for the most polarized site

$$
\delta_{\min }=\frac{1}{2}-\max _{1 \leqslant i \leqslant L}\left|\left\langle S_{i}^{z}\right\rangle\right|,
$$

whose distributions are shown at $h=3$ and $h=7$ in Fig. 1(b). There, we explicitly report two distinct trends with increasing system sizes $L$. In the ETH regime $(h=3)$, some weight is transferred with increasing $L$ toward small $-\ln \delta_{\min }$, with a peak developing at $-\ln 2$. The opposite is observed in the MBL regime $(h=7)$, where weight is moved toward large values of $-\ln \delta_{\min }$. In both cases, we qualitatively spot that rare events of the competing phase (rare insulating bottlenecks for the delocalized phase versus thermal bubbles in the MBL regime) become less relevant upon increasing $L$.

Let us now turn to a more quantitative analysis of the extreme polarization finite-size scaling. At strong disorder, assuming that the magnetizations along the chain are independently drawn from the distribution $P\left(m_{z}\right)$ of Fig. 1(a), the deviation from perfect polarization verifies $\int_{0}^{\delta_{\min }} P(x) \mathrm{d} x \sim$ $1 / L$ [82], which for a power-law tail, Eq. (3), yields

$$
\delta_{\min }(L) \sim L^{-\gamma}
$$

Such a scaling has dramatic consequences since for any $\gamma \neq$ 0 , we expect $\delta_{\min }(L) \rightarrow 0$ when $L \rightarrow \infty$, meaning spin freezing and therefore chain breaking at the thermodynamic limit.

Extreme polarizations: Numerical results. In order to numerically check the power-law scaling Eq. (5) and probe the chain breaking mechanism at the microscopic level, the most polarized site is recorded for each finite-length sample. Typical values are computed from shift-invert ED simulations at infinite temperature, and disorder averaging is performed over a large number of realizations, at least $10^{3}$ samples. Results as a function of chain lengths $L$ are shown in Fig. 2(a) for a wide range of disorder strengths. As expected from the extreme value argument Eq. (5), at strong disorder we observe a power-law decay with $L$, indicating a chain breaking at the thermodynamic limit. In contrast, for weak disorder $\delta_{\text {min }}$ does not vanish with $L$, but instead tends to $1 / 2$, showing "healing" in a way similar to Kane-Fisher physics $[83,84]$. Such 


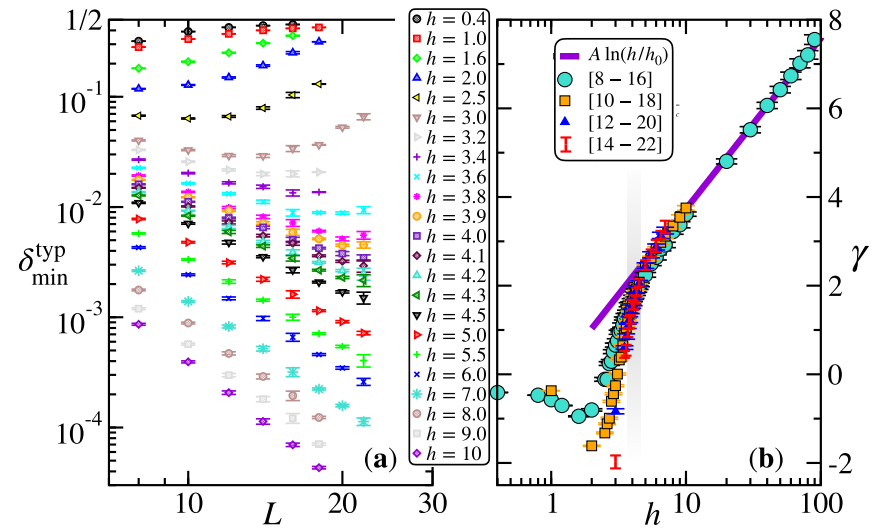

FIG. 2. (a) Typical deviation $\delta_{\min }^{\mathrm{typ}}$ Eq. (4), plotted against system size $L$ for a wide regime of disorder $h$. Log-log scale reveals the power-law decay Eq. (5) at large enough $h$, while $\delta_{\min }^{\mathrm{typ}} \rightarrow 1 / 2$ at weak disorder. (b) Freezing exponent $\gamma$ governing the decay. Various symbols stand for four different fitting windows including five points in the range $\left[L_{\min }, L_{\max }\right]$. At large disorder, the exponent grows logarithmically as $\gamma=A \ln \left(h / h_{0}\right)$ with fitting parameters $A=1.67(5)$, $h_{0} \approx 1$. The gray-shaded area shows the critical region.

radically different behaviors correspond to the two phases of the model, as we argue below.

In Fig. 2(b), we show the $h$ dependence of the freezing exponent $\gamma$ obtained from power-law fits to the form Eq. (5) over four different fitting windows. Finite-size scaling toward perfect polarization is governed by a disorder-dependent freezing exponent $\gamma$, which shows a logarithmic divergence at large $h$, a behavior explained below by analytical arguments.
Conversely, at weak disorder we observe a finite-size (possibly nonmonotonous, see also Ref. [85] for similar effects) crossover, and ultimately $\gamma \rightarrow 0$ as expected from the ETH. Before making a quantitative scaling analysis of ED data (Fig. 3), we first provide an analytical understanding of the chain-breaking mechanism.

Analytical derivation at large disorder. Using the JordanWigner transformation, we can rewrite Eq. (1) as interacting spinless fermions in a random potential

$$
\mathcal{H}=\sum_{i}\left[\frac{1}{2}\left(c_{i}^{\dagger} c_{i+1}+c_{i+1}^{\dagger} c_{i}\right)-h_{i} n_{i}\right]+\sum_{i} n_{i} n_{i+1} .
$$

The first sum, describing free fermions, can be diagonalized as $\mathcal{H}_{0}=\sum_{k=1}^{L} \mathcal{E}_{k} b_{k}^{\dagger} b_{k}$ with new fermionic operators $b_{k}=$ $\sum_{i=1}^{L} \phi_{i}^{k} c_{i}$. The single-particle orbitals $\phi_{i}^{k}$ are exponentially localized for any $h \neq 0$, and the interacting Hamiltonian is rewritten in this "Anderson basis" as four terms:

$$
\begin{aligned}
\mathcal{H} & =\sum_{k}\left(\mathcal{E}_{k}+\mathcal{V}_{k}^{(1)}\right) n_{k}+\sum_{k \neq l} \mathcal{V}_{k, l}^{(2)} n_{k} n_{l} \\
& +\sum_{k \neq l \neq m} \mathcal{V}_{k, l, m}^{(3)} n_{k} b_{l}^{\dagger} b_{m}+\sum_{k \neq l \neq m \neq n} \mathcal{V}_{k, l, m, n}^{(4)} b_{k}^{\dagger} b_{l}^{\dagger} b_{m} b_{n} .
\end{aligned}
$$

In the strong disorder limit $h \gg 1$, the second line can be neglected [81]. The number operators $n_{k}$ then form a complete basis of local operators commuting with $\mathcal{H}$ and among themselves: In other words, the Hamiltonian is written in $l$-bit form [55,68,70,86-88], and the $n_{k}$ are good approximation of the $l$ bits in the strong disorder limit [89]. The fermion density and thus the spin polarization at real-space position $i$ is then simply the sum of contributions coming from occupied
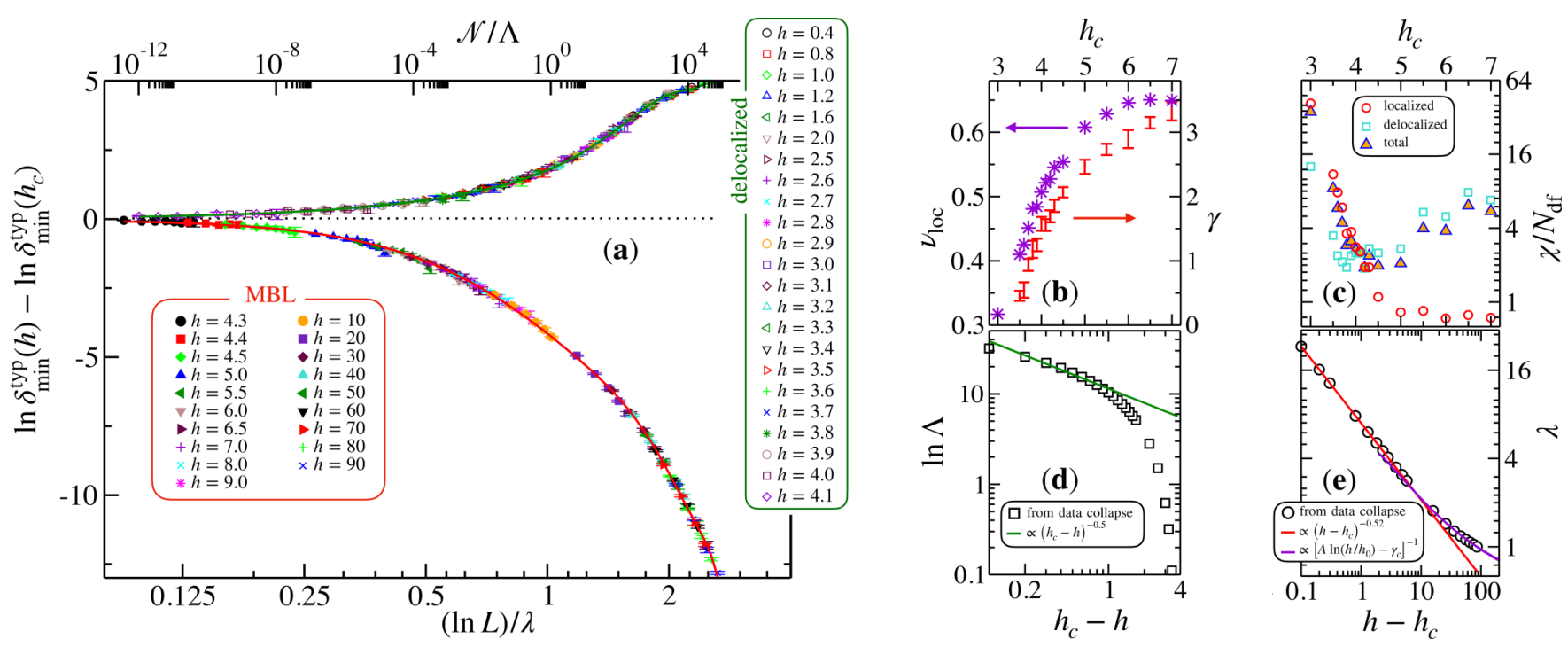

FIG. 3. (a) Scaling plots for both delocalized (top) and MBL (bottom) regimes following Eq. (10) with $h_{c}=4.2$. Green and red curves show the scaling functions $f$ and $g$ of Eq. (10) obtained from Taylor expansions close to $h_{c}$ fitted to the data [81,91,92] with two distinct (disorder-dependent) scaling parameters: $\Lambda$ and $\lambda$ for the two phases. (d) The nonergodicity volume $\Lambda$ diverges exponentially at criticality with an exponent compatible with $\nu_{\mathrm{d}}=0.5$ (green line). (e) The MBL scaling parameter $\lambda$ diverges at criticality as a power law (red line) with an exponent $v_{\text {loc }}=0.52(3)$, while there is a logarithmic dependence at strong disorder (violet line) showing perfect agreement with Fig. 2(b) using $A=1.67, h_{0}=1$, and $\gamma_{c}=1.7$. Panels (b) and (c) display the outcome of such scaling procedures obtained for different choices of $h_{c}$. In panel (b), the MBL exponent $\nu_{\text {loc }}$ smoothly increases with $h_{c}$, while in panel (c) the total $\chi$-squared statistic for the best fit $\chi$ divided by the number of degrees of freedom $N_{\mathrm{df}}$ (see text and Ref. [81]) displays an $O(1)$ minimum at $h_{c}=4.2$, where $v=0.52$. The freezing exponent $\gamma$ (red symbols) from Fig. 2(b) is also shown in panel (b), and we get $\gamma_{c}=1.7(1)$. 
orbitals:

$$
\left\langle S_{i}^{z}\right\rangle+1 / 2=\left\langle n_{i}\right\rangle=\sum_{k \text { occupied }}\left|\phi_{i}^{k}\right|^{2} .
$$

Building on previous ideas [90], we are now ready to understand the spin-freezing mechanism. If we approximate the Anderson orbitals by simple exponential functions $\left|\phi_{i}^{k}\right|^{2} \propto$ $\exp \left(-\frac{\left|i-i_{0}^{k}\right|}{\zeta_{0}}\right)$, where $i_{0}^{k}$ is the localization center of orbital $k$ and $\zeta_{0} \sim(\ln h)^{-1}$ is the noninteracting localization length at strong disorder [90], we expect the maximal density $\left\langle n_{i}\right\rangle_{\max }$ to occur in the middle of the longest region of $\ell_{\max }$ consecutive sites that are occupied by an orbital. At half-filling, a configuration with $\ell$ consecutively occupied sites appears with probability $2^{-\ell}$, which for a finite chain of length $L \gg 1$ yields $\ell_{\max } \approx \ln L / \ln 2$.

The most polarized site $i_{0}$ corresponds to the site with maximal $n_{i_{0}} \approx 1$ (resp. minimal $n_{i_{0}} \approx 0$ ) fermionic density, yielding $\left\langle S_{i_{0}}^{z}\right\rangle \approx+1 / 2$ (resp. $\left\langle S_{i_{0}}^{z}\right\rangle \approx-1 / 2$ ). As a result, the sum of exponentials Eq. (8) gives $\delta_{\min } \sim \exp \left(-\ell_{\max } /\left(2 \zeta_{0}\right)\right)$, which naturally leads to the power-law decay Eq. (5) with a freezing exponent

$$
\gamma\left(\zeta_{0}\right) \approx \frac{1}{2 \zeta_{0} \ln 2}
$$

At large disorder, numerical data perfectly agree with a logarithmic growth $\gamma(h) \propto \ln h$ [Fig. 2(b)], which validates our analytical description of the freezing mechanism. Within this description, $1 / \gamma$ is identified with the localization length of $l$ bits deep in the bulk of the largest nonthermal region. Being far away from rare thermal inclusions, the inverse freezing exponent provides an estimate of the typical $l$-bit extension, i.e., the typical localization length $\zeta: 1 / \gamma \sim \zeta$. As we further elaborate below, this has decisive consequences for our understanding of the critical behavior. Already in Fig. 2(b), when the transition is approached from the MBL side, a singular behavior develops for $\gamma(h)$ close to $h_{c}$, in agreement with Eq. (2), followed by a jump to zero in the ergodic phase. We now provide an explicit description of this critical behavior.

Scaling analysis: KT behavior. We have performed a very careful analysis of our ED data for $\delta_{\min }^{\text {typ }}(L, h)$ in order to address the transition between ergodic and MBL phases. The best data scalings [81], shown in Fig. 3(a), are obtained using two distinct scaling functions:

$$
\ln \left[\frac{\delta_{\min }^{\mathrm{typ}}(L, h)}{\delta_{\min }^{\mathrm{typ}}\left(L, h_{c}\right)}\right]= \begin{cases}f(\mathcal{N} / \Lambda) & \text { if } h<h_{c}, \\ g\left(\frac{\ln L}{\lambda}\right) & \text { if } h>h_{c} .\end{cases}
$$

In the delocalized phase $\left(h<h_{c}\right)$, we obtain a volumic scaling, in agreement with our recent multifractality analysis [36] (see also Ref. [45] for the Anderson transition on random graphs) and with ETH predictions [76], given that the density of state scales with Hilbert space size. The scaling variable is the ratio between the Hilbert space size $\mathcal{N} \approx 2^{L} / \sqrt{L}$ and a disorder-dependent nonergodicity volume $\Lambda$, which diverges exponentially at criticality: $\ln \Lambda \sim\left(h_{c}-h\right)^{-v_{\mathrm{d}}}$ with $v_{\mathrm{d}} \approx 0.5$; see Fig. 3(d). The way the freezing exponent $\gamma \rightarrow 0$ in the ergodic regime (see Fig. 2) is also controlled by $\mathcal{N} / \Lambda$.

Here the fit of the scaling function to the data is equally good for $h_{c}=3.8$ and $h_{c}=4.2$, as visible in Fig. 3(c) where the $\chi$-squared statistic divided by the number of degrees of freedom of the fit $\chi^{2} / N_{\mathrm{df}}$ is minimum [81].

In contrast, the MBL regime $\left(h>h_{c}\right)$ is best described by the function $g\left(\frac{\ln L}{\lambda}\right)$ displayed in Fig. 3(a). This is a direct consequence (see Refs. [36,49,81]) of the power-law decay Eq. (5), observed at criticality and in the MBL phase, which yields an MBL scaling function $g \propto(\ln L) / \lambda$ for large $\ln L \gg$ $\lambda$. As an outcome, the scale $\lambda$ is directly related to the freezing exponent, and therefore with the typical localization length, such that

$$
1 / \lambda \propto \gamma_{c}-\gamma \propto \zeta_{c}^{-1}-\zeta^{-1}
$$

As $h_{c} \geqslant 4.5$ increases, the goodness of fit for the MBL scaling improves; see Fig. 3(c). Indeed, by definition the logarithmic scaling perfectly describes data with algebraic behavior such as $\delta_{\min }^{\text {ty }}$ in the MBL phase. To correctly estimate $h_{c}$, we therefore need to sum the goodness of fit from both ergodic and MBL regimes; see Fig. 3(c). Then a bootstrap analysis of the data [81] gives a critical disorder strength $h_{c}=4.2(5)$. This quite large relative error of $\approx 10 \%$ reflects the difficulties inherent to this ergodic-MBL transition.

However, this very number is not decisive for our theoretical understanding of the critical point [81]. As shown for $h_{c}=4.2$ in Fig. 3(e), we get the following singularity for the inverse typical localization length,

$$
\gamma_{c}-\gamma \propto\left(h-h_{c}\right)^{\nu_{\mathrm{loc}}}
$$

with $v_{\text {loc }}=0.52(3)$ after a bootstrap analysis, and $\gamma_{c}=1.7(1)$ [see Fig. 3(b)]. This behavior quantitatively agrees with the recently proposed $[66,67] \mathrm{KT}$ mechanism; see Eq. (2). Moreover, the scaling variable $(\ln L) / \lambda$ implies that finite-size effects in real-space are formally controlled by an exponentially diverging length scale $\exp (\lambda) \sim \exp \left(h-h_{c}\right)^{-v_{\text {loc }}}$, thus confirming a KT scenario, compatible with the Harris bound. This behavior contrasts with the multifractal behavior discussed in Ref. [36], controlled by a different length scale which probes the most delocalized regions (the thermal bubbles).

Before concluding, one should note that the scaling that we propose is unusual and it is not excluded that a different finite-size scaling may also be valid in the vicinity of the transition. Nevertheless, our assumptions have been quantitatively checked against all our numerical data.

Consequences and discussion. Our key result is that the extreme value statistics in the MBL regime gives a direct access to the typical localization length $\zeta$ and therefore the typical $l$-bit extension of the MBL states. Maximally polarized sites correspond to entanglement bottlenecks: Nearly frozen spins are almost disentangled from the rest of the system, with an entanglement entropy cutting such sites given by $S=-\delta \ln \delta$ to leading order [93]. Consequently, $\delta$ controls the leading eigenvalues of the entanglement spectrum, whose observed power-law distribution $[94,95]$ is also governed by the freezing exponent $\gamma$.

Our analysis further indicates that the entire MBL regime, including the critical point, witnesses a chain-breaking mechanism at the thermodynamic limit, with finite-size effects controlled by a power-law behavior, Eq. (5). The powerlaw exponent, related to the typical localization length $\gamma \propto$ $\zeta^{-1}$, diverges logarithmically at strong disorder and displays 
a jump at the transition, with a singular critical behavior Eq. (12) in perfect agreement with a KT mechanism, Eq. (2).

Besides probing the transition universality class, spin freezing has deep consequences for MBL physics. First, it provides a simple picture accounting for the absence of thermalization at the thermodynamic limit. Another important aspect concerns the recently discussed Hilbert-space fragmentation [96-99] of the MBL regime, which here is expected to naturally emerge from spin freezing upon increasing system sizes. In addition, our results are very encouraging for the development of a perturbative decimation method [100] discarding the strongly polarized sites. Coupled to exact methods, this could provide quantitative results at strong disorder for system sizes much larger than currently accessible and thus potentially useful beyond one dimension.
Note added. Recently, we became aware of a numerical study [101] which gives indications in favor of a KT transition, based on standard observables (e.g., entanglement entropy, level statstics). Note also the recent RG work [102], which suggests that the MBL transition is in a universality class that is slightly different from KT.

Acknowledgments. This work is supported by the French National Research Agency (ANR) under Projects THERMOLOC ANR-16-CE30-0023-02, MANYLOK ANR-18CE30-0017, and GLADYS ANR-19-CE30-0013. We gratefully acknowledge F. Alet and M. Dupont for collaborations on related works. Our numerical calculations strongly benefited from the HPC resources provided by CALMIP (Grants No. 2018-P0677 and No. 2019-P0677) and GENCI (Grant No. 2018-A0030500225).
[1] P. Jacquod and D. L. Shepelyansky, Phys. Rev. Lett. 79, 1837 (1997).

[2] I. V. Gornyi, A. D. Mirlin, and D. G. Polyakov, Phys. Rev. Lett. 95, 206603 (2005).

[3] D. M. Basko, I. L. Aleiner, and B. L. Altshuler, Ann. Phys. 321, 1126 (2006).

[4] M. Žnidarič, T. Prosen, and P. Prelovšek, Phys. Rev. B 77, 064426 (2008).

[5] A. Pal and D. A. Huse, Phys. Rev. B 82, 174411 (2010).

[6] J. H. Bardarson, F. Pollmann, and J. E. Moore, Phys. Rev. Lett. 109, 017202 (2012).

[7] R. Nandkishore and D. A. Huse, Ann. Rev. Cond. Matt. 6, 15 (2015).

[8] J. Z. Imbrie, J. Stat. Phys. 163, 998 (2016).

[9] D. A. Abanin and Z. Papić, Ann. Phys. 529, 1700169 (2017).

[10] F. Alet and N. Laflorencie, C. R. Phys. 19, 498 (2018).

[11] D. A. Abanin, E. Altman, I. Bloch, and M. Serbyn, Rev. Mod. Phys. 91, 021001 (2019).

[12] M. Schreiber, S. S. Hodgman, P. Bordia, H. P. Lüschen, M. H. Fischer, R. Vosk, E. Altman, U. Schneider, and I. Bloch, Science 349, 842 (2015).

[13] J.-y. Choi, S. Hild, J. Zeiher, P. Schauß, A. Rubio-Abadal, T. Yefsah, V. Khemani, D. A. Huse, I. Bloch, and C. Gross, Science 352, 1547 (2016)

[14] J. Smith, A. Lee, P. Richerme, B. Neyenhuis, P. W. Hess, P. Hauke, M. Heyl, D. A. Huse, and C. Monroe, Nat. Phys. 12, 907 (2016).

[15] A. M. Kaufman, M. E. Tai, A. Lukin, M. Rispoli, R. Schittko, P. M. Preiss, and M. Greiner, Science 353, 794 (2016).

[16] A. Lukin, M. Rispoli, R. Schittko, M. E. Tai, A. M. Kaufman, S. Choi, V. Khemani, J. Léonard, and M. Greiner, Science 364, 256 (2019).

[17] P. Roushan, C. Neill, J. Tangpanitanon, V. M. Bastidas, A. Megrant, R. Barends, Y. Chen, Z. Chen, B. Chiaro, A. Dunsworth, A. Fowler, B. Foxen, M. Giustina, E. Jeffrey, J. Kelly, E. Lucero, J. Mutus, M. Neeley, C. Quintana, D. Sank, A. Vainsencher, J. Wenner, T. White, H. Neven, D. G. Angelakis, and J. Martinis, Science 358, 1175 (2017).

[18] K. Xu, J.-J. Chen, Y. Zeng, Y.-R. Zhang, C. Song, W. Liu, Q. Guo, P. Zhang, D. Xu, H. Deng, K. Huang, H. Wang, X.
Zhu, D. Zheng, and H. Fan, Phys. Rev. Lett. 120, 050507 (2018).

[19] B. Chiaro, C. Neill, A. Bohrdt, M. Filippone, F. Arute, K. Arya, R. Babbush, D. Bacon, J. Bardin, R. Barends, S. Boixo, D. Buell, B. Burkett, Y. Chen, Z. Chen, R. Collins, A. Dunsworth, E. Farhi, A. Fowler, B. Foxen, C. Gidney, M. Giustina, M. Harrigan, T. Huang, S. Isakov, E. Jeffrey, Z. Jiang, D. Kafri, K. Kechedzhi, J. Kelly, P. Klimov, A. Korotkov, F. Kostritsa, D. Landhuis, E. Lucero, J. McClean, X. Mi, A. Megrant, M. Mohseni, J. Mutus, M. McEwen, O. Naaman, M. Neeley, M. Niu, A. Petukhov, C. Quintana, N. Rubin, D. Sank, K. Satzinger, A. Vainsencher, T. White, Z. Yao, P. Yeh, A. Zalcman, V. Smelyanskiy, H. Neven, S. Gopalakrishnan, D. Abanin, M. Knap, J. Martinis, and P. Roushan, arXiv:1910.06024.

[20] M. Ovadia, D. Kalok, I. Tamir, S. Mitra, B. Sacépé, and D. Shahar, Sci. Rep. 5, 13503 (2015).

[21] A. De Luca and A. Rosso, Phys. Rev. Lett. 115, 080401 (2015).

[22] K. X. Wei, C. Ramanathan, and P. Cappellaro, Phys. Rev. Lett. 120, 070501 (2018).

[23] D. J. Luitz, N. Laflorencie, and F. Alet, Phys. Rev. B 91, 081103(R) (2015).

[24] F. Pietracaprina, N. Macé, D. J. Luitz, and F. Alet, SciPost Phys. 5, 045 (2018).

[25] T. Devakul and R. R. P. Singh, Phys. Rev. Lett. 115, 187201 (2015).

[26] E. V. H. Doggen, F. Schindler, K. S. Tikhonov, A. D. Mirlin, T. Neupert, D. G. Polyakov, and I. V. Gornyi, Phys. Rev. B 98, 174202 (2018).

[27] R. K. Panda, A. Scardicchio, M. Schulz, S. R. Taylor, and M. Žnidarič, EPL 128, 67003 (2020).

[28] J. Suntajs, J. Bonca, T. Prosen, and L. Vidmar, arXiv: 1905.06345.

[29] T. Chanda, P. Sierant, and J. Zakrzewski, Phys. Rev. B 101, 035148 (2020).

[30] D. A. Abanin, J. H. Bardarson, G. De Tomasi, S. Gopalakrishnan, V. Khemani, S. A. Parameswaran, F. Pollmann, A. C. Potter, M. Serbyn, and R. Vasseur, arXiv:1911.04501. 
[31] P. Sierant, D. Delande, and J. Zakrzewski, Phys. Rev. Lett. 124, 186601 (2020).

[32] J. A. Kjall, J. H. Bardarson, and F. Pollmann, Phys. Rev. Lett. 113, 107204 (2014).

[33] F. Pietracaprina, V. Ros, and A. Scardicchio, Phys. Rev. B 93, 054201 (2016).

[34] S. Schiffer, J. Wang, X.-J. Liu, and H. Hu, Phys. Rev. A 100, 063619 (2019).

[35] H.-Z. Xu, S.-Y. Zhang, Z.-Y. Rao, Z. Zhou, G.-C. Guo, and M. Gong, arXiv:1910.06601.

[36] N. Macé, F. Alet, and N. Laflorencie, Phys. Rev. Lett. 123, 180601 (2019).

[37] R. Abou-Chacra, D. J. Thouless, and P. W. Anderson, J. Phys. C: Solid State Phys. 6, 1734 (1973).

[38] A. D. Mirlin and Y. V. Fyodorov, Phys. Rev. Lett. 72, 526 (1994).

[39] C. Monthus and T. Garel, J. Phys. A 42, 075002 (2008).

[40] G. Biroli, A. Ribeiro-Teixeira, and M. Tarzia, arXiv:1211.7334.

[41] A. De Luca, B. L. Altshuler, V. E. Kravtsov, and A. Scardicchio, Phys. Rev. Lett. 113, 046806 (2014).

[42] K. S. Tikhonov, A. D. Mirlin, and M. A. Skvortsov, Phys. Rev. B 94, 220203(R) (2016).

[43] K. S. Tikhonov and A. D. Mirlin, Phys. Rev. B 94, 184203 (2016).

[44] G. Biroli and M. Tarzia, Phys. Rev. B 96, 201114(R) (2017).

[45] I. García-Mata, O. Giraud, B. Georgeot, J. Martin, R. Dubertrand, and G. Lemarié, Phys. Rev. Lett. 118, 166801 (2017).

[46] G. Biroli and M. Tarzia, arXiv:1810.07545.

[47] V. E. Kravtsov, B. L. Altshuler, and L. B. Ioffe, Ann. Phys. (Amsterdam, Neth.) 389, 148 (2018).

[48] K. S. Tikhonov and A. D. Mirlin, Phys. Rev. B 99, 024202 (2019).

[49] I. García-Mata, J. Martin, R. Dubertrand, O. Giraud, B. Georgeot, and G. Lemarié, Phys. Rev. Res. 2, 012020 (2020).

[50] M. Tarzia, Phys. Rev. B 102, 014208 (2020).

[51] A. B. Harris, J. Phys. C: Solid State Phys. 7, 1671 (1974).

[52] A. Chandran, C. R. Laumann, and V. Oganesyan, arXiv: 1509.04285 .

[53] Note that the very applicability of this bound has been criticized by C. Monthus in Ref. [103].

[54] R. Vosk and E. Altman, Phys. Rev. Lett. 110, 067204 (2013).

[55] D. A. Huse, R. Nandkishore, and V. Oganesyan, Phys. Rev. B 90, 174202 (2014).

[56] D. Pekker, G. Refael, E. Altman, E. Demler, and V. Oganesyan, Phys. Rev. X 4, 011052 (2014).

[57] R. Vasseur, A. C. Potter, and S. A. Parameswaran, Phys. Rev. Lett. 114, 217201 (2015).

[58] E. Altman and R. Vosk, Ann. Rev. Cond. Matt. 6, 383 (2015).

[59] R. Vosk, D. A. Huse, and E. Altman, Phys. Rev. X 5, 031032 (2015).

[60] A. C. Potter, R. Vasseur, and S. A. Parameswaran, Phys. Rev. X 5, 031033 (2015).

[61] P. T. Dumitrescu, R. Vasseur, and A. C. Potter, Phys. Rev. Lett. 119, 110604 (2017).

[62] M. Schiro and M. Tarzia, Phys. Rev. B 101, 014203 (2020).

[63] T. Thiery, F. Huveneers, M. Müller, and W. De Roeck, Phys. Rev. Lett. 121, 140601 (2018).

[64] T. Thiery, M. Müller, and W. De Roeck, arXiv:1711.09880.
[65] A. Goremykina, R. Vasseur, and M. Serbyn, Phys. Rev. Lett. 122, 040601 (2019).

[66] P. T. Dumitrescu, A. Goremykina, S. A. Parameswaran, M. Serbyn, and R. Vasseur, Phys. Rev. B 99, 094205 (2019).

[67] A. Morningstar and D. A. Huse, Phys. Rev. B 99, 224205 (2019).

[68] M. Serbyn, Z. Papić, and D. A. Abanin, Phys. Rev. Lett. 111, 127201 (2013).

[69] L. Rademaker, M. Ortuño, and A. M. Somoza, Ann. Phys. 529, 1600322 (2017).

[70] J. Z. Imbrie, V. Ros, and A. Scardicchio, Ann. Phys. 529, 1600278 (2017).

[71] See Ref. [49] for a similar behavior at the Anderson transition on random graphs.

[72] V. Khemani, F. Pollmann, and S. L. Sondhi, Phys. Rev. Lett. 116, 247204 (2016).

[73] S. P. Lim and D. N. Sheng, Phys. Rev. B 94, 045111 (2016).

[74] M. Dupont and N. Laflorencie, Phys. Rev. B 99, 020202(R) (2019).

[75] M. Hopjan and F. Heidrich-Meisner, Phys. Rev. A 101, 063617 (2020).

[76] J. M. Deutsch, Phys. Rev. A 43, 2046 (1991).

[77] M. Srednicki, Phys. Rev. E 50, 888 (1994).

[78] L. D’Alessio, Y. Kafri, A. Polkovnikov, and M. Rigol, Adv. Phys. 65, 239 (2016).

[79] D. J. Luitz and Y. Bar Lev, Phys. Rev. Lett. 117, 170404 (2016).

[80] D. J. Luitz and Y. Bar Lev, Ann. Phys. 529, 1600350 (2017).

[81] See Supplemental Material at http://link.aps.org/supplemental/ 10.1103/PhysRevResearch.2.042033 for technical details.

[82] S. N. Majumdar, A. Pal, and G. Schehr, Phys. Rep. 840, 1 (2020).

[83] C. L. Kane and M. P. A. Fisher, Phys. Rev. Lett. 68, 1220 (1992).

[84] G. Lemarié, I. Maccari, and C. Castellani, Phys. Rev. B 99, 054519 (2019).

[85] J.-M. Stéphan, G. Misguich, and V. Pasquier, Phys. Rev. B 82, 125455 (2010).

[86] A. Chandran, I. H. Kim, G. Vidal, and D. A. Abanin, Phys. Rev. B 91, 085425 (2015).

[87] C. Monthus, J. Stat. Mech. (2016) 033101.

[88] L. Rademaker and M. Ortuño, Phys. Rev. Lett. 116, 010404 (2016)

[89] G. De Tomasi, F. Pollmann, and M. Heyl, Phys. Rev. B 99, 241114(R) (2019).

[90] M. Dupont, N. Macé, and N. Laflorencie, Phys. Rev. B 100, 134201 (2019).

[91] K. Slevin and T. Ohtsuki, Phys. Rev. Lett. 82, 382 (1999).

[92] A. Rodriguez, L. J. Vasquez, K. Slevin, and R. A. Römer, Phys. Rev. B 84, 134209 (2011).

[93] E. S. Sørensen, M.-S. Chang, N. Laflorencie, and I. Affleck, J. Stat. Mech.: Theory Exp. (2007) P08003.

[94] A. Samanta, K. Damle, and R. Sensarma, Phys. Rev. B 102, 104201 (2020).

[95] R. Pal and A. Lakshminarayan, arXiv:2002.00682.

[96] S. Roy, D. E. Logan, and J. T. Chalker, Phys. Rev. B 99, 220201(R) (2019).

[97] S. Roy, J. T. Chalker, and D. E. Logan, Phys. Rev. B 99, 104206 (2019). 
[98] G. De Tomasi, D. Hetterich, P. Sala, and F. Pollmann, Phys. Rev. B 100, 214313 (2019).

[99] F. Pietracaprina and N. Laflorencie, arXiv:1906.05709.

[100] F. Igloi and C. Monthus, Phys. Rep. 412, 277 (2005).
[101] J. Šuntajs, J. Bonča, T. Prosen, and L. Vidmar, Phys. Rev. B 102, 064207 (2020).

[102] A. Morningstar, D. A. Huse, and J. Z. Imbrie, Phys. Rev. B 102, 125134 (2020).

[103] C. Monthus, Entropy 18, 122 (2016). 